\title{
Dynamique des compartiments du carbone et de l'azote dans le sol cultivé en niébé et sorgho dans le système zaï en zone Nord soudanienne du Burkina Faso
}

\author{
Der SOME $^{1 *}$, Edmond HIEN ${ }^{1,2}$, Komi ASSIGBETSE ${ }^{3}$, Jean Jacques DREVON ${ }^{4}$ \\ et Dominique MASSE ${ }^{3}$ \\ ${ }^{1}$ Université de Ouagadougou, Unité de Formation et de Recherche en Science de la Vie et de la Terre \\ (UFR/SVT), 03 BP 7021 Ouagadougou 03, Burkina Faso. \\ ${ }^{2}$ IRD, UMR Eco \& Sols, 01 BP 182 Ouagadougou 01, Burkina Faso. \\ ${ }^{3}$ IRD, UMR Eco \& Sols, LEMSAT (Campus IRD-ISRA) Bel-Air, B.P. 1386 Dakar, Sénégal. \\ ${ }^{4}$ UMR Eco \& Sols; Ecologie Fonctionnelle \& Biogéochimie des Sols \& Agroécosystèmes, 2 Place Viala, \\ F34060, Montpellier, France. \\ *Auteur correspondant; E-mail: d.some@yahoo.fr ; Tél: +22678 843638 ; +22674393951
}

\section{RESUME}

La gestion appropriée des sols cultivés peut permettre un stockage de carbone, en plus de l'avantage supplémentaire du maintien du niveau de fertilité qui en résulte. L'objectif de cette étude est d'appréhender l'influence réelle de pratiques culturales sous niébé ou sorgho dans le système zaï, sur l'évolution et l'organisation des teneurs de carbone $(\mathrm{C})$ et d'azote $(\mathrm{N})$ du sol à l'échelle de la parcelle. Un dispositif factoriel en blocs de Fisher a été utilisé. Les traitements comprenant des apports de fumier seul ou associé au burkina phosphate avec exportation des résidus de cultures ont été testés. Le fractionnement granulométrique de la matière organique du sol a été utilisé, séparant trois fractions. Les résultats révèlent que la répartition de $\mathrm{C}$ et $\mathrm{N}$ dans les fractions granulométriques n'a pas été affectée par les espèces cultivées. Quelle que soit la culture, le carbone et l'azote se trouvent essentiellement sous forme de matière organique stable dans la fraction 0-20 $\mu \mathrm{m}$, soit respectivement $64 \%$ et $73 \%$ pour C et N. L'apport du fumier seul ou combiné au phosphate, entraîne une augmentation de la matière organique labile ; $38 \%$ de $\mathrm{C}$ et $31 \%$ de $\mathrm{N}$ sont stockés dans la fraction 50-2000 $\mu \mathrm{m}$. La dynamique de la matière organique du sol à l'échelle de la parcelle est plus influencée par les amendements que par les espèces cultivées.

(C) 2015 International Formulae Group. All rights reserved.

Mot clés : Matière organique, sols dégradés, légumineuse, céréale, fumier, burkina phosphate.

\section{INTRODUCTION}

La matière organique $(\mathrm{MO})$ constitue la base de la fertilité des sols notamment dégradés de la zone subsaharienne. Elle joue un rôle prépondérant dans le fonctionnement global du sol au travers de ses composantes physiques, chimiques et biologiques. Sur le plan physique, la MO intervient dans la structuration du sol. Elle développe la porosité et augmente la stabilité structurale et la capacité de rétention en eau du sol (Annabi et al., 2005; Grosbellet, 2008). Au plan biologique, en qualité de source d'énergie et de nutriment pour les microorganismes et la 
faune, elle stimule l'activité biologique du sol (Calbrix et al., 2007). Son rôle dans la fertilité chimique réside principalement dans sa fonction de stockage d'éléments minéraux et sa capacité à les rendre disponibles pour les plantes par minéralisation. Elle intervient également dans la fertilité chimique par sa fonction d'échange et de sorption liée aux propriétés des surfaces des constituants organiques et organo-minéraux (Feller, 1995). Les pratiques culturales sans restitution de la MO conduisent à une perte de la fertilité des terres et à leur abandon. C'est le cas dans la Région Nord du Burkina à forte dégradation des ressources naturelles et à forte pression démographique où les résidus de récoltes sont systématiquement exportés aux fins d'aliments de bétail et d'énergie.

La zone Nord soudanienne du Burkina Faso est en effet marquée par une expansion de sols dégradés dénudés, appelés "zippelas" (langue nationale mooré), où rien ne pousse sans une forme particulière d'intervention de l'homme. Ces sols sont caractérisés par de faibles teneurs en MO et une déstructuration de l'horizon de surface se manifestant par une compaction de cette dernière limitant sa perméabilité et augmentant le ruissellement (Zombré, 2003 ; Zougmoré et al., 2004). Face au problème de disponibilité des terres cultivables, ces sols sont récupérés et remis en culture par des techniques de réhabilitation tel que le zaï qui fait appel à un travail de sol et à des apports d'amendements organiques localisés. Cette technique de culture en poquets, appelé zaï (en mooré), est largement adoptée dans le plateau central où elle est appliquée sur environ 200000 à 300000 ha (Botoni et Reij, 2009).

De nombreuses études ont été menées sur l'impact de cette technique culturale traditionnelle sur l'évolution des propriétés chimiques de ces sols dégradés et notamment la MO du sol (Zombré, 2003 ; Sawadogo et al., 2008). Il est bien établi aujourd'hui que cette technique permet une augmentation relative du taux de la MO totale du sol. Mais, l'ensemble de ces études ont porté exclusivement sur l'aspect quantitatif c'est-àdire, une évaluation simple des teneurs en MO du sol au terme d'une à trois campagnes agricoles. L'aspect qualitatif de la MO, plus spécifiquement, la caractérisation de ses compartiments dans le sol, n'a pratiquement pas fait l'objet d'étude jusque-là. Or, les fonctions de la MO vis-à-vis des propriétés du sol sont liées à celles de ses différents compartiments, autrement, à ses différentes fractions granulométriques dans le sol (Feller et al., 2001a). Les quantités de MO stockées dans le sol dépendent beaucoup plus de l'importance de sa fraction fine notamment argileuse (Feller et al., 2001b ; Barthès et al., 2008 ; Razafimbelo et al., 2010 ). Certes, de nombreuses études portant sur l'impact des systèmes de culture sur l'évolution qualitative de la MO du sol existent, (Hien et al., 2002 ; Hien, 2004 ; Pallo et al., 2009), mais elles n’ont pas concerné spécifiquement le système zaï qui se pratique sur des sols marginaux, très dégradés. L'étude de la répartition des compartiments du carbone (C) et de l'azote (N) dans les différentes fractions granulométriques du sol, permettrait de mieux cerner l'évolution de la MO du sol dans le système zaï et d'appréhender le potentiel de ses fonctions dans la réhabilitation et le maintien de la fertilité des sols dégradés dénudés.

Par ailleurs, la qualité de la MO et sa dynamique dans le sol peuvent être influencées par les types de cultures, directement par la nature biochimique des substrats organiques contenus dans leurs résidus (Bouajila et al., 2014), et aussi indirectement par l'activité biologique du sol qu'elles induisent. Les substrats organiques à $\mathrm{C} / \mathrm{N}$ bas ou riches en cellulose ont une évolution rapide avec pour conséquence un renouvellement rapide de la $\mathrm{MO}$ et une accumulation de la fraction stable (Nicolardot et al., 2001). A l'inverse, les substrats riches en lignine ont une biodégradation difficile et un turnover lent (Bahri et al., 2008). Dans le système zaï tel que pratiqué, seules les céréales, notamment le sorgho, sont ordinairement cultivées. La culture d'une légumineuse tel que le niébé dans ce système, 
par la production d'une biomasse racinaire importante et à $\mathrm{C} / \mathrm{N}$ bas (racines fines $=17$, grosses racines=29), (Groot et al., 1998a ; 1998b) par rapport à la biomasse racinaire du sorgho à $\mathrm{C} / \mathrm{N}$ élevé $(\mathrm{C} / \mathrm{N}=64)$, (Hien, 2004), produirait une $\mathrm{MO}$ de qualité différente à même d'influencer la dynamique générale et le mode de stockage de la MO dans le sol.

La présente étude a pour objet d'appréhender, au travers de la détermination de la répartition des compartiments du $\mathrm{C}$ et de $\mathrm{N}$ dans les fractions granulométriques du sol, l'influence de la culture d'une légumineuse (niébé) et d'une céréale (sorgho) d'une part, et l'impact des différents types d'amendements apportés dans le système zaï, d'autre part, sur l'évolution de la qualité de la MO du sol.

\section{MATERIEL ET METHODES}

\section{Site expérimental}

L'étude a été conduite à Pougyango $\left(12^{\circ} 58^{\prime} \mathrm{N} ; 2^{\circ} 09^{\prime} \mathrm{W}\right)$, dans le Département de Gomponsom, Province du Passoré dans la Région Nord du Burkina Faso (Figure 1). Le climat est de type Nord Soudanien (Fontès et Guinko, 1995) avec deux saisons bien marquées : une saison sèche allant d'octobre à mai et une saison pluvieuse qui s'étend de juin à septembre. La pluviométrie moyenne annuelle est comprise entre 600 et $900 \mathrm{~mm}$. Elle présente une grande variabilité spatiale, inter annuelle et intra annuelle.

L'essai a été conduit sur un sol ferrugineux tropical lessivé induré superficiel à peu profond (CPCS, 1967), ou lixisol ferrique (FAO, 2006), avec une profondeur utile variant de 18 à $26 \mathrm{~cm}$, une structure massive et une texture de type limon argileux. Situé sur glacis pente moyenne, c'est un sol entièrement dénudé et encroûté (zipellé en mooré). L'état de surface général est de type ERO (érosion), une croûte très compacte, imperméable à l'eau.

\section{Dispositif expérimental et traitements}

L'essai a été mis en place de 2006 à 2012. Un dispositif factoriel en blocs de
Fisher a été utilisé avec trois répétitions. Les facteurs testés sont le type de culture et les amendements associés au zaï. Les répétitions sont matérialisées par trois blocs séparés par des allées de $5 \mathrm{~m}$ suivant la pente. Chaque bloc renferme deux sous blocs : un sous bloc de culture pure de niébé et un sous bloc de culture pure de sorgho. Les parcelles élémentaires ont une superficie de $20 \mathrm{~m}^{2}$ (5 m x $4 \mathrm{~m}$ ) et sont séparées par des allées de $1 \mathrm{~m}$. Quatre traitements ci-dessous énumérés, ont été mis en place et ont été appliqués aux deux cultures. Ils ont consisté au creusement ou non de zaï et à l'application ou non d'amendements dans les poquets de zaï. Les poquets, de profondeur de 10 à $15 \mathrm{~cm}$ sur un diamètre de 20 à $25 \mathrm{~cm}$, sont creusés suivant un rayonnement croisé de $0,5 \mathrm{~m}$, soit une densité de semis de $50 \mathrm{~cm}$ entre les lignes et sur les lignes. Un cordon pierreux a été aménagé en amont pour freiner la vitesse du ruissellement. Les 4 traitements réalisés sont : (1) TA = Témoin absolu (semis direct sans creusement de zaï et sans amendements) ; (2) $\mathrm{ZS}=$ Zaï simple (creusement de poquets de zaï sans apport de fumure); (3) $\mathrm{ZF}=\mathrm{Zaï}+$ fumier à $3 \mathrm{t} \mathrm{ha}^{-1}(67 \mathrm{~g} /$ poquet $)$; (4) ZFP = Zä̈ + fumier à $3 \mathrm{t} \mathrm{ha}^{-1}+$ burkinaphosphate à 2 t.ha ${ }^{-}$

1 (40 g/poquet). Il n'ya pas de restitution des résidus de récoltes. La paille et les fanes sont entièrement exportées chaque année.

\section{Fumures et cultures}

L'amendement organique utilisé est un fumier de parc de bovins. Ses caractéristiques chimiques moyennes sont : C: 147,3 g. $\mathrm{kg}^{-1}$; $\mathrm{N}: 16,7$ g.kg-1 $; \mathrm{C} / \mathrm{N}: 9,3 ; \mathrm{P}_{2} \mathrm{O}_{5}: 2,3 \mathrm{~g} \cdot \mathrm{kg}^{-1}$; $\mathrm{K}_{2} 0: 16,2$ g. $\mathrm{kg}^{-1}$; $\mathrm{MgO}: 6,7$ g.kg ${ }^{-1}$; $\mathrm{Ca} 0: 7,6$ g. $\mathrm{kg}^{-1}$.

Le Burkina phosphate, phosphate naturel de Kodjari finement broyé, a été utilisé comme amendement minéral en association avec le fumier. Selon le Projet Burkina phosphate, il présente les caractéristiques chimiques suivantes: $\mathrm{P}_{2} \mathrm{O}_{5}: 25,43 \% ; \mathrm{K}_{2} \mathrm{O}$ : 0,3\% ; $\mathrm{CaO}: 34,61 \% ; \mathrm{MgO}: 0,18 \%$; solubilité dans l'eau : 0,03\%. 
Le niébé (Vigna unguiculata (L.) Walp.), variété KVX-61-1, a été retenu comme légumineuse ; la céréale utilisée est le sorgho (Sorghum bicolor (L.)), variété ICSV 1049. Toutes les deux cultures sont des variétés améliorées mises au point par l'Institut de l'Environnement et de Recherches Agricoles (INERA, Burkina Faso).

\section{Echantillonnage du sol}

Le prélèvement des échantillons a été effectué en fin de campagne, après la récolte. Sur chaque parcelle, trois échantillons élémentaires sont prélevés suivant une diagonale de la parcelle dans l'horizon 0-15 $\mathrm{cm}$ à l'intérieur des poquets. Ils sont mélangés pour former un échantillon composite, soit au total trois échantillons par traitement correspondant aux trois répétions. Les échantillons ont été ensuite séchés à l'ombre, tamisés à $2 \mathrm{~mm}$ et conservés à température ambiante.

\section{Fractionnement granulométrique de la matière organique}

Le principe du fractionnement granulométrique repose sur la séparation mécanique des compartiments de la MO sans les dénaturer (Feller, 1979). Son utilisation se justifie par sa simplicité, sa facilité de mise en œuvre, son efficacité et son action peu dénaturante. Sa réussite est conditionnée par une bonne dispersion du sol à même de permettre une séparation optimale des fractions organiques sans les détruire ni les dénaturer. La méthode utilisée a consisté à mélanger $20 \mathrm{~g}$ de sol dans $500 \mathrm{ml}$ d'eau additionnée de $10 \mathrm{ml}$ d'hexaméthaphosphate de sodium (HMP) et d'agiter ce mélange en présence de 5 billes d'agate de $1 \mathrm{cmde}$ diamètre pendant 2 heures. Le tamisage sous l'eau sur un tamis d'ouverture $50 \mu \mathrm{m}$ permet de séparer la fraction grossière comprise entre 50 et $2000 \mu \mathrm{m}$. La suspension de sol inférieure à $50 \mu \mathrm{m}$ est tamisée ensuite, toujours sous eau, sur un tamis de $20 \mu \mathrm{m}$ pour isoler la fraction comprise entre 20 à $50 \mu \mathrm{m}$.
La suspension du sol inférieure à $20 \mu \mathrm{m}$ est transvasée dans une éprouvette et complétée à 1 L. Elle est homogénéisée par plusieurs retournements de l'éprouvette, puis une aliquote de $20 \mathrm{ml}$ est siphonnée à miprofondeur puis transvasée dans une capsule en porcelaine. Les différentes fractions sont séchées à l'étuve à $40{ }^{\circ} \mathrm{C}$, pesées et broyées pour le dosage de $\mathrm{C}$ et de $\mathrm{N}$. Le fractionnement a été effectué au Laboratoire d'Agro-écologie UMR Eco \& Sols de l'IRD à Ouagadougou.

\section{Mesures de carbone et d'azote}

Le carbone total et l'azote total ont été dosés par la méthode CHN, au moyen d'un auto analyseur élémentaire EA1112 Termo Finnigan (mesure effectuée par le Laboratoire des Moyens Analytiques (LAMA), IRD Dakar/Sénégal, certifié ISO 9001).

\section{Analyse statistique}

Une analyse de variance des données a été faite à l'aide du logiciel CoSTat 6.4 pour comparer d'une part les effets des types de cultures et d'autre part, les effets des différents types d'amendements. La séparation des moyennes a été réalisée par le test de Newman-Keuls au seuil de 5\%.

\section{RESULTATS}

\section{Masse des fractions}

Les bilans de masse du poids total de l'échantillon fractionné par rapport à la somme des poids des fractions obtenues sont compris en moyenne entre 99,96 et $100,98 \%$. De façon générale, la masse de la fraction fine 0-20 $\mu \mathrm{m}$ (argile+limon fin) est plus élevée au niveau des deux cultures et pour tous les traitements (Tableau 1). Elle est suivie de la fraction grossière sableuse $50-2000 \mu \mathrm{m}$ et de la fraction limon grossier 20-50 $\mu \mathrm{m}$. La fraction $0-20 \mu \mathrm{m}$ est plus élevée dans les témoins sans zaï (TA) que dans les traitements au zaï (ZS, ZF, ZFP). 
Contenus en carbone des fractions granulométriques

Influence des types de cultures

Les contenus en carbone de l'ensemble des trois fractions sous niébé ne sont pas différents, en quantité et en proportion, de ceux sous culture de sorgho (Tableau 2).

En termes de proportion, la fraction 0$20 \mu \mathrm{m}$ renferme la plus grande partie du carbone sous les deux cultures, en moyenne $64 \%$ sous niébé comme sous sorgho.

La fraction 50-2000 $\mu \mathrm{m}$ contient une proportion relativement importante de carbone, environ $28 \%$ du carbone du sol sous culture de niébé, et $29 \%$ sous sorgho.

La fraction 20-50 $\mu \mathrm{m}$ renferme moins de 1/10è du carbone total du sol sous les deux cultures, soit en moyenne $8 \%$ du carbone total du sol sous les deux cultures.

\section{Effet des amendements}

En fonction des traitements, il y a une variabilité de la répartition du $\mathrm{C}$ dans les fractions granulométriques du sol (Tableau 2). Mais pour l'ensemble des traitements, les contenus en $\mathrm{C}$ décroissent de la fraction fine à la fraction grossière. Au niveau du témoin sans zaï ni amendement (TA), environ $76 \%$ du $\mathrm{C}$ du sol est contenu dans la fraction 0-20 $\mu \mathrm{m}$. Moins du quart du C est reparti entre la fraction $20-50 \mu \mathrm{m}(9-10 \%)$ et la fraction 50$2000 \mu \mathrm{m}(14-15 \%)$. Il en est de même pour le traitement au zaï simple sans amendement ZS où environ $69 \%$ du $\mathrm{C}$ est contenu dans la fraction 0-20 $\mu \mathrm{m}$. Mais dans ce dernier, le contenu en carbone de la fraction 50-2000 $\mu \mathrm{m}$ est plus important, environ le double de celui du premier.

$\mathrm{Au}$ niveau des parcelles amendées au fumier simple $(\mathrm{ZF})$ et au fumier combiné au Burkina phosphate (ZFP), il n'y a pas de différence significative entre les deux traitements. Dans la fraction 0-20 $\mu \mathrm{m}$, les teneurs en $\mathrm{C}$ représentent environ $54 \%$ du $\mathrm{C}$ total du sol pour ZF et $55 \%$ avec ZFP sous les deux cultures. Au niveau de ces traitements, une importante quantité de $\mathrm{C}$ se retrouve dans la fraction sableuse $50-2000 \mu \mathrm{m}(37 \%$ pour $\mathrm{ZF}$ et environ $38 \%$ pour ZFP).
Du point de vue quantité, au niveau de la fraction 0-20 $\mu \mathrm{m}$, les teneurs en $\mathrm{C}$ des traitements ne sont pas significativement différentes $(\mathrm{P}=0,93$ pour le niébé ; $\mathrm{P}=0,68$ pour le sorgho). Il en est de même pour la fraction $20-50 \mu \mathrm{m}(\mathrm{P}=0,85$ pour le niébé; $\mathrm{P}=0,35$ pour le sorgho).

Dans la fraction grossière $50-2000 \mu \mathrm{m}$, les teneurs en $\mathrm{C}$ de ZF $\left(5,26-5,52 \mathrm{mg}^{-\mathrm{g}^{-1}}\right.$ de sol sous niébé et sorgho) et de ZFP (5,74-5.69 mg.g ${ }^{-1}$ de sol) sont significativement plus élevées ( $\mathrm{P}<0,0001)$, environ le double de celles de ZS (2,50-2,62 mg. ${ }^{-1}$ de sol) et trois fois celles de TA $\left(1,89-1,62 \mathrm{mg} \cdot \mathrm{g}^{-1} \mathrm{de}\right.$ sol$)$.

L'addition du phosphate naturel au fumier dans le zaï n'a pas d'effet significatif par rapport au fumier seul sur les teneurs en carbone total du sol quelle que soit la fraction granulométrique et le type de culture.

Contenus en azote des fractions granulométriques

Influence des types de cultures

Les résultats révèlent que la répartition de $\mathrm{N}$ du sol n'est pas influencée significativement par les types de cultures (Tableau 3). Comme le carbone, la fraction 0$20 \mu \mathrm{m}$ renferme la plus grande partie de $\mathrm{N}$ du sol et dans les mêmes proportions au niveau des deux cultures ( $73 \%$ en moyenne).

La fraction 50-2000 $\mu \mathrm{m}$ contient moins de $\mathrm{N}$ et les mêmes proportions sont également observées au niveau des deux cultures (environ $21 \%$ de l'azote total du sol).

La fraction $20-50 \mu \mathrm{m}$ renferme moins de $1 / 10^{\text {è }}$ de $\mathrm{N}$ total du sol sous les deux cultures, soit environ $6 \%$ de $\mathrm{N}$ du sol sous niébé comme sous culture de sorgho.

\section{Effet des amendements}

$\mathrm{La}$ répartition de $\mathrm{N}$ total dans les fractions granulométriques du sol est marquée par une variabilité en fonction des traitements (Tableau 3).

$\mathrm{Au}$ niveau du témoin sans zaï ni amendement (TA), l'essentiel de $\mathrm{N}$ est contenu dans la fraction $0-20 \mu \mathrm{m} ; 86$ à $87 \%$ de $\mathrm{N}$ total du sol (respectivement sous niébé et sorgho) sont retrouvés dans cette fraction. Il en est de même pour les traitements au zaï 
simple sans amendement (ZS) où plus de $3 / 4$ de $\mathrm{N}$ du sol sont stocké dans la fraction 0-20 $\mu \mathrm{m}(79 \%$ et $80 \%$ de $\mathrm{N}$ total du sol sous niébé et sorgho). Mais au niveau de ZS, en terme de proportion, le contenu en $\mathrm{N}$ du sol est plus élevé dans la fraction sableuse 50-2000 $\mu \mathrm{m}$, environ le double de celui du TA (16\% contre $7 \%$ sous sorgho et $15 \%$ contre $9 \%$ sous le niébé).

$\mathrm{Au}$ niveau des parcelles amendées au fumier simple (ZF) et au fumier combiné au Burkina phosphate (ZFP), il n'y a pas de différence significative entre les deux traitements aussi bien en termes de contenus que de proportions de $\mathrm{N}$ du sol dans les différentes fractions. En terme de proportion, environ $63 \%$ de $\mathrm{N}$ total du sol des deux traitements sont stockés dans la fraction 0-20 $\mu \mathrm{m}$ sous les deux cultures. Dans la fraction grossière 50-2000 $\mu \mathrm{m}$ sous les deux cultures, les contenus en $\mathrm{N}$ du sol au niveau de $\mathrm{ZF}$ et ZFP représentent environ 4 fois celui observé au niveau du témoin (TA) et environ 2 fois celui enregistré avec les traitements au zai simple sans amendement (ZS).

Du point de vue quantité, en culture de niébé, les teneurs en $\mathrm{N}$ total de $\mathrm{ZF}\left(0,85 \mathrm{mg}\right.$. $\mathrm{g}^{-}$ ${ }^{1}$ de sol) et de ZFP (0.91 mg.g ${ }^{-1}$ de sol) ne sont pas significativement différentes de celles du témoin TA $\left(1,01 \mathrm{mg}^{-g^{-1}}\right.$ de sol) et de ZS $(0,76$ mg. $\mathrm{g}^{-1}$ de sol) dans la fraction 0-20 $\mu \mathrm{m}$. Par contre, il y'a une différence significative entre TA et ZS. Sous culture de sorgho les teneurs en $\mathrm{N}$ sont homogènes entre tous les traitements dans cette fraction (Tableau 3).

La fraction 20-50 $\mu \mathrm{m}$ contient les plus faibles quantités de $\mathrm{N}$ du sol pour l'ensemble des traitements et au niveau des deux cultures. Du point de vue statistique, il n'y a pas de différence significative entre les traitements sous les deux cultures.

Dans la fraction 50-2000 $\mu \mathrm{m}$ les contenus en $\mathrm{N}$ total sont relativement importants mais nettement inférieurs à ceux de la fraction 0-20 $\mu \mathrm{m}$. Les teneurs en $\mathrm{N}$ de ZF (0,41-0,40 mg.g ${ }^{-1}$ de sol sous niébé et sorgho) et de ZFP (0,45-0,40 mg.g ${ }^{-1}$ de sol sous niébé et sorgho) sont significativement plus élevées, environ 4 fois plus que celui de
TA $\left(0,10-0,08 \mathrm{mg} \cdot \mathrm{g}^{-1}\right.$ de sol) et environ 3 fois plus que celui de ZS (0,14 mg. $\mathrm{g}^{-1}$ de sol).

\section{Rapport $\mathrm{C} / \mathrm{N}$ des fractions granulométriques \\ Influence des types de cultures}

Les rapports $\mathrm{C} / \mathrm{N}$ du sol ne sont pas influencés significativement par les types de cultures (Tableau 3). Au niveau des trois fractions, Il n'y a pas une différence significative entre les rapports $\mathrm{C} / \mathrm{N}$ du sol sous niébé et ceux sous culture de sorgho. Toutefois, dans les 3 fractions, les valeurs ont tendance à être plus élevées en culture de sorgho que sous celle du niébé. Au niveau des deux cultures, le rapport $\mathrm{C} / \mathrm{N}$ du sol décroit de la fraction grossière 50-2000 $\mu \mathrm{m}$ (16-17 sous niébé et sorgho) à la fraction fine 0-20 $\mu \mathrm{m}$ (9-10) (Tableau 3)

\section{Effet des amendements}

Le rapport $\mathrm{C} / \mathrm{N}$ des fractions granulométriques du sol est marqué par une variabilité en fonction des traitements (Tableau 3). Dans la fraction 0-20 $\mu \mathrm{m}$, les rapports $\mathrm{C} / \mathrm{N}$ sont les plus bas pour tous les traitements et au niveau des deux cultures. Il n'y a pas de différence significative entre les traitements au niveau des deux cultures. Les valeurs varient entre 9,07 et 10,31.

$\mathrm{Au}$ niveau de la fraction $20-50 \mu \mathrm{m}$, il y a une différence significative entre les traitements seulement au niveau de la culture du niébé $(\mathrm{P}=0,03)$. Les valeurs de $\mathrm{C} / \mathrm{N}$ du témoin sans zaï ni amendement (TA) sont significativement plus élevées que celles des parcelles amendées au fumier simple $(\mathrm{ZF})$ et au fumier combiné au phosphate (ZFP).

La fraction sableuse 50-2000 $\mu \mathrm{m}$ a les valeurs de $\mathrm{C} / \mathrm{N}$ les plus élevés. Les traitements TA et ZS ont des $\mathrm{C} / \mathrm{N}$ significativement plus élevées que ceux de ZF et ZFP sous les deux cultures. En culture de niébé, $\mathrm{C} / \mathrm{N}$ varie dans l'ordre suivant : TA $(18,6) \geq \mathrm{ZS}(17,9)>\mathrm{ZFP}$ $(13,0) \geq Z$ ZF $(12,8)$. IL varie dans le même ordre sous la culture du sorgho: TA $(20,5) \geq$ ZS $(19,3)>$ ZFP $(14,1) \geq \operatorname{ZF}(13,7)$. 
D. SOME et al. / Int. J. Biol. Chem. Sci. 9(2): 954-969, 2015
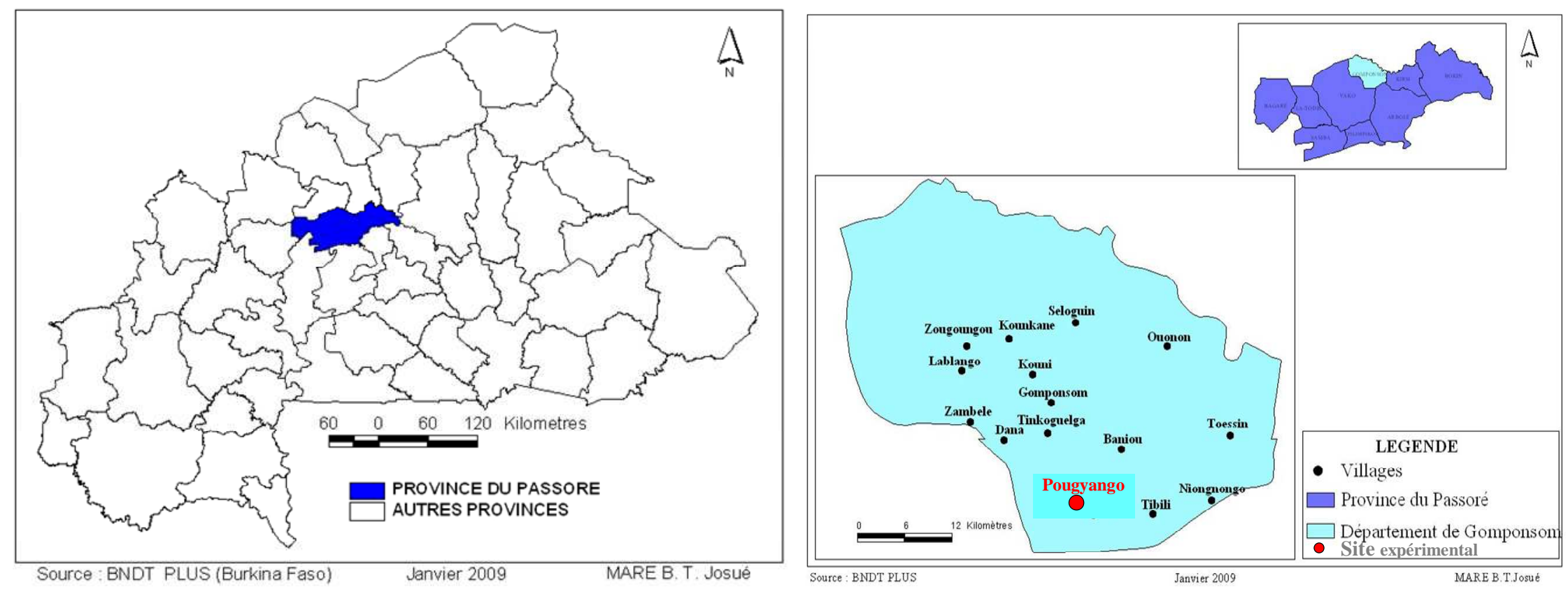

Figure 1: Localisation du site d'étude. 
D. SOME et al. / Int. J. Biol. Chem. Sci. 9(2): 954-969, 2015

Tableau 1: Bilan en masse des fractions (\% de sol).

\begin{tabular}{lcccccccc}
\hline \multirow{2}{*}{ Fractions } & \multicolumn{4}{c}{ Niébé } & \multicolumn{5}{c}{ Sorgho } \\
\cline { 2 - 8 } & TA & ZS & ZF & ZFP & TA & ZS & ZF & ZFP \\
\hline $0-20 \mu \mathrm{m}$ & 49,33 & 38,64 & 39,00 & 43,60 & 49,84 & 37,69 & 39,31 & 40,44 \\
$20-50 \mu \mathrm{m}$ & 24,94 & 22,26 & 26,23 & 21,59 & 22,07 & 25,33 & 25,71 & 23,37 \\
$50-2000 \mu \mathrm{m}$ & 25,76 & 39,19 & 34,77 & 34,77 & 28,01 & 37,16 & 35,76 & 37,17 \\
Somme & 100,03 & 100,09 & 99,98 & 99,96 & 99,92 & 100,18 & 100,79 & 100,98 \\
\hline \multicolumn{1}{c}{}
\end{tabular}

Tableau 2: Concentrations (mg.g ${ }^{-1}$ de fraction), contenus (mg. $\mathrm{g}^{-1} \mathrm{de}$ sol) et proportions (\%) en C des fractions.

\begin{tabular}{|c|c|c|c|c|c|c|c|}
\hline \multirow[t]{2}{*}{ Fractions } & \multirow[t]{2}{*}{ Traitements } & \multicolumn{2}{|c|}{$\begin{array}{c}\mathrm{C} \\
\left(\mathrm{mg}^{-1} \mathrm{de}^{-1} \text { fraction }\right)\end{array}$} & \multicolumn{2}{|c|}{$\begin{array}{c}\mathrm{C} \\
\left(\mathrm{mg}^{-1} \mathrm{~g}^{-1} \text { de sol) }\right. \\
\end{array}$} & \multicolumn{2}{|c|}{$\begin{array}{c}\mathrm{C} \\
(\% \mathrm{C} \text { sol total }) \\
\end{array}$} \\
\hline & & Niébé & Sorgho & Niébé & Sorgho & Niébé & Sorgho \\
\hline \multirow{5}{*}{$0-20 \mu \mathrm{m}$} & TA & $19,06 \mathrm{a}$ & $18,81 \mathrm{a}$ & $9,41 \mathrm{a}$ & $9,32 \mathrm{a}$ & 74,96 & 76,71 \\
\hline & $\mathrm{ZS}$ & $19,15 \mathrm{a}$ & $19,31 \mathrm{a}$ & $7,37 \mathrm{a}$ & $7,29 a$ & 68,58 & 68,55 \\
\hline & $\mathrm{ZF}$ & $19,75 \mathrm{a}$ & $20,60 a$ & $7,74 \mathrm{a}$ & $8,15 \mathrm{a}$ & 54,42 & 54,15 \\
\hline & ZFP & $19,21 \mathrm{a}$ & $20,16 a$ & $8,49 \mathrm{a}$ & $8,14 \mathrm{a}$ & 55,08 & 54,59 \\
\hline & Moyenne par culture & $19,29 A$ & $19,72 B$ & $8,37 A$ & $8,23 \mathrm{~A}$ & 63,51 & 63,50 \\
\hline \multirow{5}{*}{$20-50 \mu \mathrm{m}$} & TA & $4,75 \mathrm{a}$ & $4,48 \mathrm{a}$ & $1,24 \mathrm{a}$ & $1,20 \mathrm{a}$ & 10,01 & 9,37 \\
\hline & $\mathrm{ZS}$ & $3,80 \mathrm{a}$ & $2,65 \mathrm{a}$ & $0,77 \mathrm{a}$ & $0,65 \mathrm{a}$ & 7,50 & 6,28 \\
\hline & $\mathrm{ZF}$ & $4,21 \mathrm{a}$ & $4,96 a$ & $1,14 \mathrm{a}$ & $1,28 \mathrm{a}$ & 8,22 & 8,85 \\
\hline & ZFP & $5,04 \mathrm{a}$ & $4,66 \mathrm{a}$ & $1,11 \mathrm{a}$ & $1,06 \mathrm{a}$ & 7,40 & 7,15 \\
\hline & Moyenne par culture & $4,45 \mathrm{~A}$ & $4,19 A$ & 1,06 & 1,05 & 8,16 & 7,91 \\
\hline
\end{tabular}

961 
D. SOME et al. / Int. J. Biol. Chem. Sci. 9(2): 954-969, 2015

\begin{tabular}{|c|c|c|c|c|c|c|c|}
\hline \multirow{4}{*}{$50-2000 \mu \mathrm{m}$} & TA & $7,32 \mathrm{~b}$ & $6,53 \mathrm{~b}$ & $1,89 \mathrm{~b}$ & $1,68 \mathrm{~b}$ & 15,03 & 13,92 \\
\hline & $\mathrm{ZS}$ & $6,72 b$ & $6,98 \mathrm{~b}$ & $2,50 \mathrm{~b}$ & $2,62 \mathrm{~b}$ & 23,92 & 25,17 \\
\hline & $\mathrm{ZF}$ & $14,99 \mathrm{a}$ & $15,49 \mathrm{a}$ & $5,26 \mathrm{a}$ & $5,52 \mathrm{a}$ & 37,35 & 36,99 \\
\hline & ZFP & $16,43 a$ & $15,33 \mathrm{a}$ & $5,74 \mathrm{a}$ & $5,69 \mathrm{a}$ & 37,51 & 38,26 \\
\hline \multirow{3}{*}{ Somme des fractions } & TA & $31,13 \mathrm{~b}$ & $29,82 \mathrm{~b}$ & $12,54 \mathrm{c}$ & $12,20 \mathrm{~b}$ & & \\
\hline & $\mathrm{ZS}$ & $29,67 \mathrm{~b}$ & $28,94 \mathrm{~b}$ & $10,64 d$ & $10,56 \mathrm{~b}$ & & \\
\hline & Moyenne par culture & $35,11 A$ & $34,99 A$ & $13,16 A$ & $13,15 A$ & & \\
\hline \multicolumn{8}{|l|}{ ANOVA } \\
\hline \multirow{3}{*}{$0-20 \mu \mathrm{m}$} & $\mathrm{P}$ & 0,93 & 0,68 & 0,13 & 0,09 & & \\
\hline & Signifiance & NS & NS & NS & NS & & \\
\hline & $\mathrm{CV}$ & 7,58 & 9,78 & 11,35 & 9,45 & & \\
\hline \multirow[t]{2}{*}{$50-2000 \mu \mathrm{m}$} & Signifiance & S & S & $\mathrm{S}$ & $\mathrm{S}$ & & \\
\hline & $\mathrm{CV}$ & 9,97 & 7,04 & 14,96 & 15,17 & & \\
\hline \multirow{3}{*}{ Somme des fractions } & $\mathrm{P}$ & 0,003 & 0,006 & 0,0001 & 0,003 & & \\
\hline & Signifiance & S & $\mathrm{S}$ & $\mathrm{S}$ & $\mathrm{S}$ & & \\
\hline & $\mathrm{CV}$ & 6,61 & 9,14 & 3,71 & 6,90 & & \\
\hline
\end{tabular}

Les traitements portant la même lettre (dans les colonnes pour les traitements et les lignes pour les moyennes des cultures) ne sont pas statistiquement différents au seuil de $5 \%$;

P : probabilité ; CV : coefficient de variation; NS : non significatif ; S : significatif ; TA : Témoin absolu ; ZS : Zaï simple ; ZF : Zaï + fumier ; ZFP : Zaï + fumier + Burkina phosphate. 
D. SOME et al. / Int. J. Biol. Chem. Sci. 9(2): 954-969, 2015

Tableau 3 : Concentrations (mg. $\mathrm{g}^{-1}$ de fractions), contenus (mg. $\mathrm{g}^{-1} \mathrm{de}$ sol) et proportions (\%) en $\mathrm{N}$ et Rapport C/N des fractions.

\begin{tabular}{|c|c|c|c|c|c|c|c|c|c|}
\hline \multirow[t]{2}{*}{ Fractions } & \multirow[t]{2}{*}{ Traitements } & \multicolumn{2}{|c|}{$\begin{array}{c}\mathrm{N} \\
\left(\mathrm{mg}^{-1} \mathrm{de} \mathrm{fraction}^{-}\right.\end{array}$} & \multicolumn{2}{|c|}{$\begin{array}{c}\mathrm{N} \\
\left(\mathrm{mg} \cdot \mathrm{g}^{-1} \text { de sol) }\right.\end{array}$} & \multicolumn{2}{|c|}{$\begin{array}{c}\mathrm{N} \\
(\% \text { sol total })\end{array}$} & \multicolumn{2}{|c|}{$\mathbf{C} / \mathbf{N}$} \\
\hline & & Niébé & Sorgho & Niébé & Sorgho & Niébé & Sorgho & Niébé & Sorgho \\
\hline \multirow{5}{*}{$0-20 \mu \mathrm{m}$} & TA & $2,06 a$ & $2,00 \mathrm{a}$ & $1,01 \mathrm{a}$ & $0,99 \mathrm{a}$ & 85,65 & 86,90 & $9,39 \mathrm{a}$ & $9,47 a$ \\
\hline & $\mathrm{ZS}$ & $2,02 \mathrm{a}$ & $1,88 \mathrm{a}$ & $0,76 \mathrm{~b}$ & $0,70 \mathrm{a}$ & 79,80 & 79,40 & $9,62 \mathrm{a}$ & $10,31 \mathrm{a}$ \\
\hline & $\mathrm{ZF}$ & $2,18 \mathrm{a}$ & $2,13 \mathrm{a}$ & $0,85 \mathrm{ab}$ & $0,84 \mathrm{a}$ & 62,77 & 62,51 & $9,07 \mathrm{a}$ & $9,68 \mathrm{a}$ \\
\hline & ZFP & $2,07 \mathrm{a}$ & $2,08 \mathrm{a}$ & $0,91 \mathrm{ab}$ & $0,84 \mathrm{a}$ & 63,21 & 63,30 & $9,26 \mathrm{a}$ & $9,73 \mathrm{a}$ \\
\hline & Moyenne par culture & $2,08 A$ & $2,02 A$ & $0,88 A$ & $0,84 A$ & 72,86 & 73,02 & 9,33 & 9,80 \\
\hline \multirow{5}{*}{$20-50 \mu \mathrm{m}$} & TA & $0,27 \mathrm{a}$ & $0,27 \mathrm{a}$ & $0,07 \mathrm{a}$ & $0,07 \mathrm{a}$ & 5,69 & 5,85 & $17,44 \mathrm{a}$ & $17,41 \mathrm{a}$ \\
\hline & $\mathrm{ZS}$ & $0,23 \mathrm{a}$ & $0,17 \mathrm{a}$ & $0,05 \mathrm{a}$ & $0,04 \mathrm{a}$ & 5,18 & 4,88 & $14,99 \mathrm{ab}$ & $15,10 \mathrm{a}$ \\
\hline & $\mathrm{ZF}$ & $0,33 \mathrm{a}$ & $0,37 \mathrm{a}$ & $0,09 a$ & $0,09 \mathrm{a}$ & 6,60 & 7,21 & $12,83 \mathrm{~b}$ & $13,44 \mathrm{a}$ \\
\hline & ZFP & $0,38 \mathrm{a}$ & $0,34 \mathrm{a}$ & $0,08 \mathrm{a}$ & $0,08 \mathrm{a}$ & 5,89 & 5,99 & $12,98 \mathrm{~b}$ & $13,42 \mathrm{a}$ \\
\hline & Moyenne par culture & $0,30 A$ & $0,29 A$ & $0,072 A$ & $0,071 \mathrm{~A}$ & 5,840539 & 5,984991 & 14,56 & 14,84 \\
\hline \multirow{5}{*}{$50-2000 \mu \mathrm{m}$} & TA & $0,40 \mathrm{~b}$ & $0,32 \mathrm{~b}$ & $0,10 \mathrm{~b}$ & $0,08 \mathrm{~b}$ & 8,66 & 7,25 & $18,57 \mathrm{a}$ & $20,54 a$ \\
\hline & $\mathrm{ZS}$ & $0,38 \mathrm{~b}$ & $0,36 \mathrm{~b}$ & $0,14 b$ & $0,14 \mathrm{~b}$ & 15,02 & 15,72 & $17,88 \mathrm{a}$ & $19,28 \mathrm{a}$ \\
\hline & $\mathrm{ZF}$ & $1,17 \mathrm{a}$ & $1,13 \mathrm{a}$ & $0,41 \mathrm{a}$ & $0,40 \mathrm{a}$ & 30,63 & 30,28 & $12,79 \mathrm{~b}$ & $13,73 \mathrm{~b}$ \\
\hline & ZFP & $1,28 \mathrm{a}$ & $1,09 \mathrm{a}$ & $0,45 \mathrm{a}$ & $0,40 \mathrm{a}$ & 30,90 & 30,71 & $12,98 \mathrm{~b}$ & $14,06 \mathrm{~b}$ \\
\hline & Moyenne par culture & $0,81 A$ & $0,73 A$ & $0,27 A$ & $0,26 A$ & 21,30 & 20,99 & 15,56 & 16,90 \\
\hline \multirow{5}{*}{$\begin{array}{l}\text { Somme des } \\
\text { fractions }\end{array}$} & TA & $2,73 \mathrm{~b}$ & $2,59 \mathrm{~b}$ & $1,18 \mathrm{a}$ & $1,14 \mathrm{a}$ & & & & \\
\hline & $\mathrm{ZS}$ & $2,63 \mathrm{~b}$ & $2,41 \mathrm{~b}$ & $0,95 \mathrm{~b}$ & $0,88 \mathrm{~b}$ & & & & \\
\hline & $\mathrm{ZF}$ & $3,68 \mathrm{a}$ & $3,63 \mathrm{a}$ & $1,35 \mathrm{a}$ & $1,33 \mathrm{a}$ & & & & \\
\hline & ZFP & $3,73 \mathrm{a}$ & $3,51 \mathrm{a}$ & $1,44 \mathrm{a}$ & $1,32 \mathrm{a}$ & & & & \\
\hline & Moyenne par culture & $3,19 A$ & $3,03 A$ & $1,23 A$ & $1,17 A$ & & & & \\
\hline
\end{tabular}


D. SOME et al. / Int. J. Biol. Chem. Sci. 9(2): 954-969, 2015

\begin{tabular}{|c|c|c|c|c|c|c|c|}
\hline ANOVA & & & & & & & \\
\hline \multirow{3}{*}{$0-20 \mu \mathrm{m}$} & $\mathrm{P}$ & 0,80 & 0,65 & 0,03 & 0,08 & 0,90 & 0,22 \\
\hline & Signifiance & NS & NS & $\mathrm{S}$ & NS & NS & NS \\
\hline & $\mathrm{CV}$ & 10,25 & 12,03 & 8,43 & 12,67 & 10,03 & 4,53 \\
\hline \multirow{3}{*}{$20-50 \mu \mathrm{m}$} & $\mathrm{P}$ & 0,29 & 0,15 & 0,05 & 0,30 & 0,03 & 0,06 \\
\hline & Signifiance & NS & NS & NS & NS & $\mathrm{S}$ & NS \\
\hline & $\mathrm{CV}$ & 30,33 & 33,30 & 19,43 & 41,83 & 10,34 & 10,68 \\
\hline \multirow{3}{*}{$50-2000 \mu \mathrm{m}$} & $\mathrm{P}$ & 0,0002 & 0,0000 & 0,0006 & 0,0001 & 0,001 & 0,006 \\
\hline & Signifiance & $\mathrm{S}$ & $\mathrm{S}$ & $\mathrm{S}$ & $\mathrm{S}$ & $\mathrm{S}$ & $\mathrm{S}$ \\
\hline & $\mathrm{CV}$ & 16,48 & 8,56 & 21,23 & 16,90 & 7,05 & 10,26 \\
\hline \multirow{3}{*}{$\begin{array}{l}\text { Somme des } \\
\text { fractions }\end{array}$} & $\mathrm{P}$ & 0,01 & 0,008 & 0,006 & 0,01 & & \\
\hline & Signifiance & $\mathrm{S}$ & $\mathrm{S}$ & S & $\mathrm{S}$ & & \\
\hline & CV & 11,04 & 10,90 & 8,81 & 10,21 & & \\
\hline
\end{tabular}




\section{DISCUSSION}

Dans la pratique du zä̈, la fumure organique (fumier, compost) est apportée en début de saison dans les poquets et recouverte d'une mince couche de terre issue de l'excavation des poquets ; ceci afin de limiter les actions du vent ou du ruissellement qui pourrait déplacer ces apports. Au fil de la saison culturale, les poquets se remplissent progressivement de sol issu de l'entraînement de la terre excavée des cuvettes qui se trouvent en aval. En général, à la fin de la saison de culture, les cuvettes sont totalement comblées. La matière organique (MO) contenue dans les poquets en fin de campagne a pour origine, principalement la fumure apportée en début de saison, la MO résiduelle contenue dans la terre d'excavation, la biomasse racinaire des cultures de la campagne en cours, et accessoirement des débris organiques divers piégés au cours de la saison. Le fractionnement granulométrique a permis de séparer trois fractions correspondant aux sables (50-2000 $\mu \mathrm{m})$, au limon grossier $(20-50 \mu \mathrm{m})$ et à l'argile + limon fin (0-20 $\mu \mathrm{m})$. Ces fractions présentent des masses et des contenus en carbone (C) et azote $(\mathrm{N})$ variables.

\section{Influence des cultures et des traitements sur les contenus en carbone $(C)$ et en azote (N) des fractions}

Les résultats révèlent que les teneurs en $\mathrm{C}$ et en $\mathrm{N}$ et leur répartition dans l'ensemble des fractions granulométriques ne sont pas significativement différents entre la culture du niébé et celle du sorgho.

Du point de vue répartition, quel que soit le traitement (avec ou sans zaï et avec ou sans amendement), les contenus en $\mathrm{C}$ et en $\mathrm{N}$ décroissent de la fraction fine $(0-20 \mu \mathrm{m})$ à la fraction grossière sableuse $(50-2000 \mu \mathrm{m})$ sous les deux cultures. Cette forme de répartition de $\mathrm{C}$ et $\mathrm{N}$ dans les fractions corrobore les résultats obtenus par certains auteurs pour la culture de sorgho en zone agroclimatique et sur des formations pédologiques similaires au site d'étude (Hien, 2004 ; Pallo et al., 2009).
La prédominance de $\mathrm{C}$ et $\mathrm{N}$ dans la fraction fine traduisant une dominance de la MO stable par rapport à la fraction libre, peut être liée essentiellement à la texture du sol qui renferme des taux d'argile+limon assez élevés, variant de 35 à 44\%. Il est montré en effet que les teneurs en MO dépendent essentiellement de la texture du sol aussi bien en situations cultivées que non cultivées. Elles sont élevées dans les sols à texture argileuse et faibles dans les sols sableux (Feller et al., 2001b ; Barthès et al., 2008). La MO stable dépend en particulier des teneurs en argile et en limon, fractions minérales avec lesquelles elle forme des complexes organo-minéraux stables et où elle est protégée contre la dégradation (Baldock et Skjemstad, 2000). Ce qui explique que dans ces sols aux faibles apports d'amendements organiques et sans restitution des résidus de récoltes (pailles et des fanes), la fraction granulométrique fine est le lieu privilégié de stockage de la matière organique du sol. Par contre, dans les fractions grossières $50-2000 \mu \mathrm{m}$ et $20-50 \mu \mathrm{m}$, la matière organique composée essentiellement de débris végétaux et fongiques libres à des degrés divers de décomposition, est celle qui est facilement minéralisée et dont le stock est renouvelée annuellement.

A l'inverse de l'effet obtenu avec les types de cultures, la répartition du carbone et de l'azote dans les différentes fractions a été affectée par les traitements appliqués aux parcelles. Au niveau des témoins sans zaï ni amendement (TA) où le sol n'a pas subi de perturbation depuis des décennies, environ 75 à $77 \%$ du carbone total et 86 à $87 \%$ de l'azote total du sol sont contenus dans la fraction 0-20 $\mu \mathrm{m}$. La MO du sol se trouve donc principalement sous forme stable, liée aux particules minérales. Cela se justifie par l'absence quasi totale d'apport exogène de MO. Les apports dus aux racines sont quasi inexistantes ou extrêmement faibles pour permettre un renouvellement de la MO libre. Dans les parcelles traitées au zaï sans amendement (ZS), les contenus en $\mathrm{C}$ et $\mathrm{N}$ ont augmenté en termes de quantité et de 
proportion par rapport à TA dans la fraction sableuse $(50-2000 \mu \mathrm{m})$. Cette augmentation est liée principalement aux apports en biomasse racinaire des cultures et accessoirement aux débris divers piégés dans les poquets de zaï. A l'inverse, au niveau de la fraction $0-20 \mu \mathrm{m}$, les teneurs en $\mathrm{C}$ et $\mathrm{N}$ ont baissé par rapport à TA. Le creusement des poquets entraîne une augmentation de l'aération, une amélioration de l'humidité du sol et une déprotection de la MO liée, favorisation sa minéralisation. Ce qui pourrait expliquer la diminution des teneurs en $\mathrm{C}$ et $\mathrm{N}$ observée dans la fraction fine au niveau de ZS. En système de culture zaï, on ne dispose pas de données dans la littérature à titre illustratif, mais de façon générale, il est établi que le travail du sol augmente la minéralisation de la MO. Bayer et al. (2002) et Grandière et al. (2007), rapportent que les contenus en $\mathrm{C}$ des fractions $<50 \mu \mathrm{m}$ sont plus élevés en culture sans travail de sol qu'en culture avec travail de sol. Par ailleurs, il est relaté dans la littérature que les cultures sans restitution des résidus ni apport d'amendement entraînent une diminution de la MO du sol (Hien et al., 2002).

L'addition du Burkina phosphate au fumier (ZFP) n'a pas influencé significativement la répartition de $\mathrm{C}$ et $\mathrm{N}$ dans les différentes fractions granulométriques, suggérant que la combinaison du phosphate au fumier n'a pas d'effet significatif sur la production de la biomasse racinaire des cultures par rapport au fumier seul. Au niveau de ces deux traitements (ZF et ZFP), les teneurs en $\mathrm{C}$ et $\mathrm{N}$ dans la fraction fine $(0-20$ $\mu \mathrm{m})$ ne différent pas de celles du témoin TA et du traitement ZS. L'apport du fumier seul ou additionné au Burkina phosphate n'a pas influencé significativement les quantités de la MO stable du sol. Par contre, l'augmentation des contenus en $\mathrm{C}$ et $\mathrm{N}$ dans la fraction sableuse (50-2000 $\mu \mathrm{m})$, traduit une augmentation de la fraction de MO labile du sol due au fumier et à la biomasse racinaire plus importante produite par les cultures. Ces résultats vont dans le même sens que ceux relatés dans la littérature en système de culture ordinaire. Hien (2004) a observé une importante augmentation des teneurs de $\mathrm{C}$ dans la fraction grossière en culture de sorgho due à l'apport de fumier. Cette augmentation serait imputable à l'amélioration de la production de la biomasse racinaire.

\section{Effet des cultures et des traitements sur les $\mathrm{C} / \mathrm{N}$ des fractions}

Comparés au sorgho, les rapports $\mathrm{C} / \mathrm{N}$ des fractions en culture du niébé ne sont pas différents du point de vue statistique. Sous les deux cultures, les rapports $\mathrm{C} / \mathrm{N}$ décroissent de la fraction grossière sableuse (16 sous niébé et 17 sous sorgho) à la fraction fine argilolimoneuse (9 sous niébé et 10 sous sorgho). La décroissance du rapport $\mathrm{C} / \mathrm{N}$ de la fraction grossière à la fraction fine est couramment observée en zone tropicale (Hien, 2004 ; Pallo et al., 2009) comme en zone tempérée (Christensen, 2001). Cela s'expliquerait selon Feller (1995) et Christensen (2001) par la nature et les caractéristiques de la matière organique associée aux fractions. Les fractions grossières sableuses $(>50 \mu \mathrm{m})$ constituées de débris végétaux peu décomposés riches en $\mathrm{C}$, ont des rapports $\mathrm{C} / \mathrm{N}$ élevés. Les fractions limoneuses, avec des contenus en MO moyens constituées de fragments végétaux et fongiques à décomposition avancée, ont des rapports $\mathrm{C} / \mathrm{N}$ moyens. Tandis que les fractions fines argileuses constituées de débris végétaux et fongiques très décomposés et principalement de MO amorphes associés à des résidus microbiens (fraction 0-20), ont des rapports $\mathrm{C} / \mathrm{N}$ plus faibles.

$\mathrm{Au}$ niveau des deux cultures, la variation des rapports $\mathrm{C} / \mathrm{N}$ entre les traitements apparaît principalement au niveau des fractions grossières. Dans la fraction fine (0-20), les rapports $\mathrm{C} / \mathrm{N}$ sont homogènes pour tous les traitements. Ils varient entre 9,3 et 9,6 sous niébé et entre 9,5 et 10,3 sous sorgho. Ces valeurs corroborent bien celles relatées dans la littérature, notamment celles obtenues par Hien (2004) en culture de sorgho dans la 
même zone Agroclimatique. Grandière et al. (2007) ont observé que les rapports $\mathrm{C} / \mathrm{N}$ des fractions ne sont pas affectés par le mode de gestion du sol. Cette situation s'explique par le fait que dans la fraction fine, quel que soit le traitement, la MO est caractérisée par un taux d'humification plus élevé et une part importante d'origine microbienne, plus humidifiée et plus riche en N. La fraction grossière est, par contre, marquée par une importante variation des rapports $\mathrm{C} / \mathrm{N}$ entre les traitements. Les témoins sans amendements (TA, ZS), ont les rapports $\mathrm{C} / \mathrm{N}$ plus élevés que les traitements au fumier $(\mathrm{ZF}$ et ZFP). Au niveau du TA l'absence totale d'apport de fumure depuis des décennies a entraîné un appauvrissement en MO des fractions grossières. La MO qui y est présente est celle qui est résistante à la dégradation, riche en $\mathrm{C}$ et pauvre en azote ; ce qui explique le rapport $\mathrm{C} / \mathrm{N}$ plus élevé. Il en est de même pour ZS où les faibles apports de MO dus principalement à la biomasse racinaire n'ont pas modifié significativement les contenus en $\mathrm{C}$ et $\mathrm{N}$ de la fraction grossière par rapport à TA. En revanche, avec les traitements $\mathrm{ZF}$ et ZFP, il y a eu une importante augmentation de $\mathrm{MO}$ riche en $\mathrm{N}$ dans la fraction grossière due au fumier et à la biomasse racinaire plus importante produite par les cultures; ce qui justifie les rapports $\mathrm{C} / \mathrm{N}$ plus faibles.

\section{Conclusion}

Les résultats de cette étude révèlent que l'utilisation d'une légumineuse comme le niébé dans le système zaï sur sol ferrugineux tropical induré peu profond avec exportation totale des résidus de cultures ne semble pas influencer à court terme la répartition de $\mathrm{C}$ et $\mathrm{N}$ dans les fractions granulométriques du sol par rapport à la culture céréalière, notamment du sorgho. Au niveau des deux cultures, la plus grande partie de $\mathrm{C}$ et $\mathrm{N}$ du sol est stockée dans la fraction argilo-limoneuse sous forme de MO stable.

Les apports organiques dus à l'amendement au fumier simple ou associé au phosphate naturel entraînent une augmentation sensible de la MO labile liée à la fraction sableuse, mais sont sans influence apparente sur l'évolution de la MO stable du sol.

Dans les conditions agropédologiques de Pougyango, le maintien d'un niveau convenable de MO stable ne peut être envisagé sous culture continue de niébé ou de sorgho que si les pratiques culturales allient amendements organiques et fertilisation minérale, notamment le burkina phosphate. La pratique culturale est bien une variable explicative des teneurs et stocks de $\mathrm{C}$ du sol.

\section{REMERCIEMENTS}

Les auteurs remercient le Projet FABATROPIMED de la Fondation Agropolis qui a soutenu financièrement les travaux. Ils sont reconnaissants à Moussa BARRY, Prosper SAWADAGO, techniciens au Laboratoire d'agro-écologie, UMR Eco \& Sols de l'IRD Ouagadougou et à Abdoulaye DABRE, stagiaire à l'UMR Eco \& Sols de l'IRD Ouagadougou pour leur assistance technique.

\section{REFERENCES}

Annabi M, Le Bissonnais Y, Francou C, Le Villio-Poitrenaud M, Houot S. 2005. Utilisation de composts pour améliorer la stabilité structurale des sols limoneux. Echo. $M O$, 56: 3-8.

Bahri H, Rasse DP, Rumpel C, Dignac MF, Bardoux G, Mariotti A. 2008. Lignin degradation during a laboratory incubation followed by ${ }^{13} \mathrm{C}$ isotope analysis. Soil Biology \& Biochemistry, 40: 1916-1922.

Baldock JA, Skjemstad JO. 2000. Role of the soil matrix and minerals in protecting natural organic materials against biological attack. Organic Geochemistry, 31: 697-710.

Barthès BG, Kouakoua E, Larré-Larrouy MC, Razafimbelo TM, De Luca EF, Azontonde A, Neves CSVJ, De Freitas PL, Feller CL. 2008. Texture and sesquioxide effects on water-stable 
aggregates and organic matter in some tropical soils. Geoderma, 143: 14-25.

Bayer C, Dick DP, Ribeiro GM, Scheuermann KK. 2002. Carbon stocks in organic matter fractions as affected by land use and soil management, with emphasis on no-tillage effect. Ciência Rural, Santa Maria, 32(3): 401-406.

Botoni E, Reij C. 2009. La transformation silencieuse de l'environnement et des systèmes de production au Sahel : Impacts des investissements publics et privés dans la gestion des ressources naturelles. CILSS, 61p.

Bouajila K, Ben Jeddi F, Taamallah H, Jedidi N, Sanaa M. 2014. Effets de la composition chimique et biochimique des résidus de cultures sur leur décomposition dans un sol Limono-Argileux du semi aride. J. Mater. Environ. Sci., 5(1): 159166.

Calbrix R, Barray S, Chabrerie O, Laetitia Fourrie L, Laval K. 2007. Impact of organic amendments on the dynamics of soil microbial biomass and bacterial communities in cultivated land. Applied Soil Ecology, 35: 511-522.

Christensen BT. 2001. Physical fractionation of soil and structural and functional complexity in organic matter turnover. European Journal of Soil Science, 52: 345-353.

CPCS. 1967. Classification des Sols. Travaux de la Commission de Pédologie et de Cartographie des Sols (1963-1967). ENSA-Grignon, Laboratoire de Pédologie- Géologie : Paris ; 96.

FAO. 2006. World reference base for soil resources. World Soil Resources Reports $\mathrm{N}^{\circ}$ 103, FAO, Rome, 128 p.

Feller C. 1979. Une méthode de fractionnement granulométrique de la matière organique des sols application aux sols tropicaux, à textures grossières, très pauvres en humus. Cah. ORSTOM, Sér. Pedol., 17(4): 339-346.

Feller C. 1995. La matière organique dans les sols tropicaux à argile 1: 1. Recherche de compartiments organiques fonctionnels. Une approche granulométrique. Thèse et documents microfichés, Université Louis Pasteur, Strasbourg, France. 393p.

Feller C, Balesdent J, Nicolardot B, Cerri C. 2001a. Approaching "Functional" Soil Organic Matter Pools through ParticleSize Fractionation: Examples for Tropical Soils. In Assesment Methods for Soil Carbon, Lal R, Kimble KM, Follet RF, Stewart BA (eds). CRC Press LLC: Boca Raton, USA; 53-67.

Feller C, Albrecht A, Blanchart E, Cabidoche YM, Chevallier T, Hartmann C, Eschenbrenner V, Larré-Larrouy MC, Ndandou JF. 2001b. Soil organic carbon sequestration in tropical areas. General considerations and analysis of some edaphic determinants for Lesser Antilles soils. Nutrient Cycling in Agroecosystem, 61: 19-31.

Fontes J, Guinko S. 1995. Carte de la végétation et de l'occupation du sol du Burkina Faso. Notice explicative. Ministère de la Coopération Française, projet Campus, 67 p.

Grandière I, Razafimbelo $\mathrm{T}$, Barthès $\mathrm{B}$, Blanchart E, Louri J, Ferrer H, Chenu C, Wolf N, Albrecht A, Feller C. 2007. Distribution granulo-densimétrique de la matière organique dans un sol argileux sous semis direct avec couverture végétale des Hautes Terres malgaches. Etude et Gestion des Sols, 14(2): 117- 133.

Groot JJR, Traoré M, Koné D. 1998a. Description du système racinaire de trois espèces fourragères en zone soudanosahélienne : Andropogon gayanus, Vigna unguiculata et Stylosanthes hamata. Biotechnol. Agron. Soc. Environ., 2: 106119.

Groot JJR, Soumaré A, Koné D. 1998b. Dynamique de la matière organique du sol. In L'Intensification Agricole au Sahel, Henk B, Keffing S (eds). Kartala; 243-263. 
Grosbellet Claire. 2008. Evolution et effet sur la structuration du sol de la matière organique apportée en grande quantité. Thèse de doctorat. Université d'Angers, France, $210 \mathrm{p}$.

Hien E, Ganry F, Hien V, Oliver R. 2002. Dynamique du carbone dans un sol de savane du Sud-Ouest Burkina sous l'effet de la mise en culture et des pratiques culturales. In : Savanes africaines : des espaces en mutation, des acteurs face à de nouveaux défis, Jamin JY, Seiny Boukar L, Floret C (ed). Actes du colloque, mai 2002, Garoua, Cameroun ; 27-31.

Hien. 2004. Dynamique du carbone dans un Acrisol ferrique du Centre Ouest Burkina: Influence des pratiques culturales sur le stock et la qualité de la matière organique. Thèse de doctorat, Ecole Nationale Supérieure Agronomique de Montpellier, France, 118 p.

Nicolardot B, Recous S, Mary B. 2001. Simulation of $\mathrm{C}$ and $\mathrm{N}$ mineralization during crop residue decomposition: A simple dynamic model based on the C:N ratio of the residues. Plant and Soil, 228: 83-103.

Pallo JPP, Sedogo MP, Sawadogo N, Zombré NP. 2009. Statut de la matière organique des sols de la zone Nord soudanienne au Burkina Faso. Biotechnol. Agron. Soc. Environ., 13(1): 139-142.
Razafimbelo T, Albrecht A, Feller C, Ravelojaona H, Moussa N, Razanamparany C, Rakotoarinivo C, Razafintsalama H, Michellon R, Naudin K, Rabeharisoa L. 2010. Stockage de carbone dans les sols sous systèmes de culture en semis direct sous couvert végétal $(\mathrm{SCV})$ dans différents contextes pédoclimatiques à Madagascar. Etude et Gestion des Sols, 17 (2): 143-158.

Sawadogo H, Bock L, Lacroix D, Zombré NP. 2008. Restauration des potentialités de sols dégradés à l'aide du zaï et du compost dans le Yatenga (Burkina Faso). Biotechnol. Agron. Soc. Environ., 12(3): 279-290.

Zombré NP. 2003. Les sols très dégrades "zipella" du centre nord du Burkina Faso: dynamique, caractéristiques morpho-biopédologiques et impacts des techniques de restauration. Thèse de doctorat d'Etat, Université de Ouagadougou, $327 \mathrm{p}$.

Zougmoré R, Ouattara K, Mando A, Ouattara B. 2004. Rôle des nutriments dans le succès des techniques de conservation des eaux et des sols (cordons pierreux, bandes enherbées, zaï et demi-lunes) au Burkina Faso. Sécheresse, 15 (1): 41-48. 